\title{
An automated runway and Skinner box apparatus'
}

\section{P. V. HANFORD, INDIANA UNIVERSITY DOWNTOWN CAMPUS J. ZIMMERMAN AND W. R. LECKRONE, INDIANA UNIVERSITY MEDICAL SCHOOL}

An automated runway is described which permits animals to be run without handling between trials. The runway consists of three legs of a square with a Skinner box forming the base of the square. Results showing the stability of running performance on CRF are presented.

Two of the most frequently used instruments for examining "learning" or "motivational" variables in the animal laboratory are the runway and the Skinner box. Although both instruments have been used to assess the behavioral effects of similar variables, results of allegedly similar studies have not always been in agreement (i.e., see Amsel, 1964). One might argue that differences in results obtained with the two instruments could be accounted for by differences in behaviors generated and in behavioral measures used. It is also quite possible, however, that differences in the two sets of results might be accounted for, at least in part, by differences in the nature of the operating procedures employed with the two instruments. Whereas most studies with the Skinner box examine free operant behavior in individual organisms in a completely automated environment, studies with the runway employ large groups of animals and discrete trial procedures. Furthermore, the $E$ intervenes manually at several stages of each trial by dropping a $S$ into a start box, opening the start door, and removing the $S$ from the goal box. These manual procedures can readily lead to $\mathrm{E}$ bias and subjectivity, thereby influencing the nature of the results.

To eliminate the use of manual procedures, we have designed an automated runway which permits the automatic programming of reinforcement contingencies and the automatic recording of results obtained both in the runway and in a Skinner box. The apparatus is pictured in Fig. 1. It consists of a three-legged runway which forms three sides of a square and a Skinner box, one wall of which forms the base of the square. Each leg of the runway is $2 \mathrm{ft}$ in length and the Skinner is $2 \mathrm{ft}$ long by $1 \mathrm{ft}$ wide. Both the runway and the Skinner box are covered with Plexiglas. Entrance into and exit from the runway are controlled by two doors (white $\mathrm{D}$ and black $\mathrm{D}$, respectively) located at opposite ends of the Skinner box. These doors are arranged so that $S$ can only travel through the apparatus in a clockwise direction. Access to the alley is controlled by a solenoid lock on the door (black D) leading into the alley. Lights located at both ends of the box (white Cs) and over the alley (black Cs) provide cues for appropriate responses. A bar (A) and a liquid magazine dispenser
(B) are located on the front wall of the box adjacent to the door (white D) through which $S$ enters the box from the runway. Photocells located in the runway (1 and 4) can be used to record running time in the apparatus. Measures of running speed can be obtained by using interruption of the photocells in conjunction with a bar press or with the termination of a magazine cycle. The programming of contingencies and the recording of results is carried out with standard electromechanical programming equipment used in operant research. The equipment is housed in a control room adjacent to the room in which this apparatus is placed. Visual access to the animal's performance is provided by a one-way vision mirror mounted between the two rooms.

Data from a study in which Ss were reinforced on a continuous reinforcement schedule are presented below to illustrate the stability of performance which can be obtalned with this apparatus.

Four male albino rats, maintained at $80 \%$ of freefeeding weight, were trained to run through the apparatus in three steps. Each was first trained to press the bar in the Skinner box by making chocolate milk reinforcement contingent on each response. Second, each was trained to run through the alley in a clockwise fashion, by placing it in the alley adjacent to the locked entrance door (black D) and reinforcing a bar press after re-entry into the Skinner box. Finally, each $S$ was placed in the Skinner box and trained to enter the alley by opening the entry door (black D).

Each $\mathrm{S}$ was placed in the Skinner box at the beginning of a session and not handled until the end of

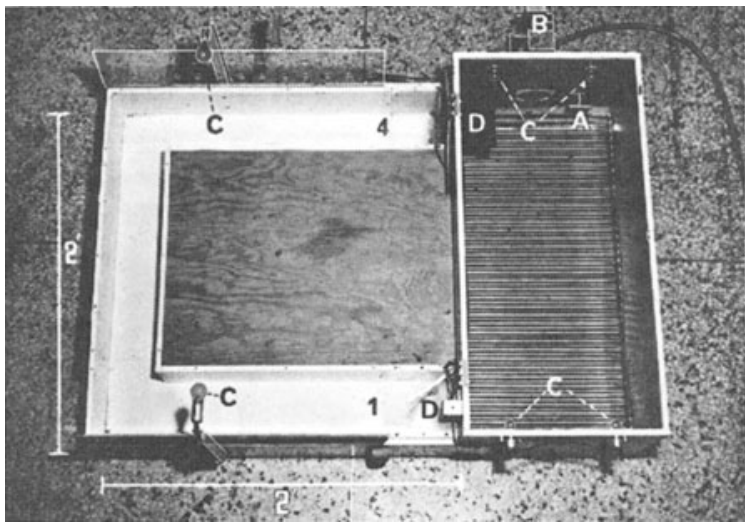

Fig. 1. The runway and Skinner box apparatus. A-bar; B-liquid reinforcement magazine; $C$-lights used as $\mathrm{s}^{\mathrm{D}} \cdot \mathrm{s}$ in box and runway; D-doors (both are in open position); 1 and 4-photocells mounted in the outer walls of the runway and located 6 in. from the alley entry door and box entry door, respectively. 


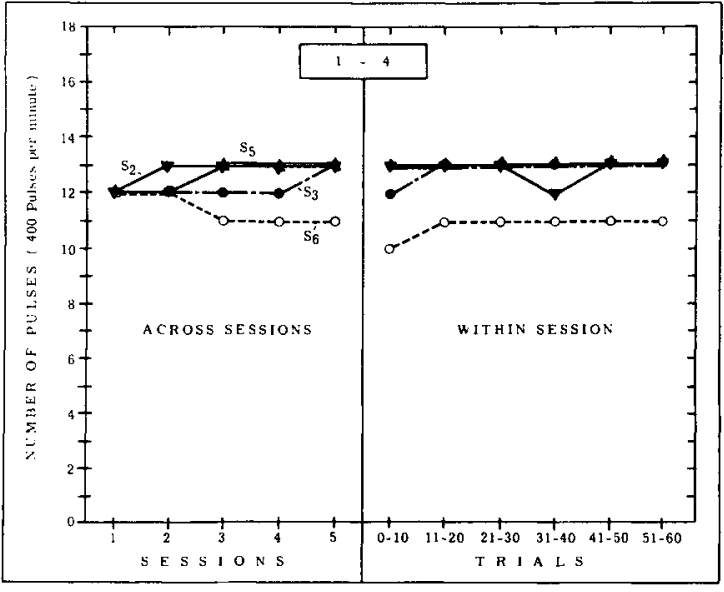

Fig. 2. Running times from Photocell 1 to Photocell 4. Points in the left panel represent the median number of pulses for each of the five final sessions under continuous reinforcement. Points in the right panel represent the median number of pulses in each successive block of 10 trials over the final session.

the session, 60 trials. A trial started with the illumination of the two lights (in the Skinner box) adjacent to the exit door and the two lights over the alley. The door leading into the alley (black D) was concurrently unlocked. S pushed this door open, entered the alley, and tripped Photocells 1 and 4 as it passed them. When $S$ tripped the latter cell the lights previously illuminated went off and the lights over the magazine were turned on. When $S$ reached the end of the alley it pushed open the door, entered the box, and pressed the bar. A bar press raised the food dipper and turned the lights off. At the end of the magazine cycle the lights at the far end of the box and over the alley came on again signalling the start of the next trial. Three measures of running speed were obtained in each trial by using a three-channel print-out counter, each head of which was driven during an appropriate period of time by a pulsing electronic timer. The starting time was measured between the end of a magazine cycle and the interruption of Photocell 1. The running time was measured between the interruption of Photocells 1 and 4 . Finally the goal approach time was measured between the interruption of Photocell 4 and the bar press.

Figure 2 describes the final performances obtained for running speed in the alley (Photocells 1-4) for each of the four Ss. Each point plotted in the left half of the figures represents the median value of the measure in a given session. The five successive points for each $S$ are derived from the final five sessions to which it was exposed (each was exposed for approximately 65 daily sessions). The data plotted in the right half of each figure present the median values of the measure obtained in each successive block of 10 trials during the final session. Figure 2 shows that in the case of each $S$, running time was extremely consistent both across the final five sessions and within the final session.

The above data indicate that this instrument may be a useful tool for studying the kinds of variables which have been investigated with the runway. It offers the advantages of the Skinner box in reducing the potential influence of the E's bias on the animal's performance while also eliminating the tedium of manually running the animals. In addition, it broadens the scope of the runway by permitting either a discrete trial or a free response trial procedure to be used.

\section{Reference}

Amsel, A., Mackinnon, J. R., Rashotte, M. E., \& Surridge, C. T. Partial reinforcement (acquisition effects within subjects). $J$. exp. Anal. Behav., 1964, 7, 135-138.

\section{Note}

1. This study was supported in part by a Grant in Aid from The Graduate School, Indiana University. 